\title{
Diatomáceas (Bacillariophyceae) de sedimentos superficiais dos reservatórios em cascata do Rio Paranapanema (SP/PR, Brasil): Coscinodiscophyceae e Fragilariophyceae
}

\author{
Luciane Fontana $^{1}$ e Denise de C. Bicudo ${ }^{2,3}$
}

Recebido: 16.04.2009; aceito: 10.06.2009

ABSTRACT - (Diatoms (Bacillariophyceae) from superficial sediments of cascade reservoirs in the Paranapanema River (SP/ PR, Brazil): Coscinodiscophyceae and Fragilariophyceae). Floristic survey of Coscinodiscophyceae and Fragilariophyceae from superficial sediments of cascade reservoirs in the Paranapanema River is presented. Sediment samplings were gathered with dredge in three sampling stations of four reservoirs located in the upper Paranapanema River (Jurumirim and Chavantes reservoirs) and middle portion (Salto Grande and Capivara reservoirs), and in two sampling stations in the lower river (Rosana reservoir). Thirteen infrageneric taxa were identified, six of them belonging to class Coscinodiscophyceae and the other seven to Fragilariophyceae. Thalassiosira rudis Tremarin et al., Asterionella formosa Hass. var. formosa, Staurosirella martyi (Herib.) Morales \& Manoylov, Staurosirella subcapitata (Freng.) Morales and Staurosira cf. acutirostrata Metz. \& L.-B. were new records for the Paranapanema River Basin, and this was the second record of T. rudis worldwide.

Key words: algae, flora, reservoir, sediment

RESUMO - (Diatomáceas (Bacillariophyceae) de sedimentos superficiais dos reservatórios em cascata do Rio Paranapanema (SP/PR, Brasil): Coscinodiscophyceae e Fragilariophyceae). Foi realizado inventário das diatomáceas pertencentes às classes Coscinodiscophyceae e Fragilariophyceae de sedimentos superficiais dos reservatórios em cascata do Rio Paranapanema. Amostras de sedimentos foram coletadas com draga, em três estações de amostragem de quatro reservatórios localizados nas regiões do alto trecho do rio (represas de Jurumirim e Chavantes) e médio (represas de Salto Grande e Capivara) e, em duas estações, no baixo Paranapanema (Represa de Rosana). Foram identificados 13 táxons infragenéricos, seis e sete respectivamente pertencentes às classes Coscinodiscophyceae e Fragilariophyceae. As espécies Thalassiosira rudis Tremarin et al., Asterionella formosa Hass. var.formosa, Staurosirella martyi (Herib.) Morales \& Manoylov, Staurosirella subcapitata (Freng.) Morales e Staurosira cf. acutirostrata Metz. \& L.-B. constituem novas citações para a bacia hidrográfica do rio Paranapanema, sendo este o segundo registro de T. rudis em âmbito mundial.

Palavras-chave: algas, flora, reservatório, sedimentos

\section{Introdução}

Estudos referentes à diatomoflora de ambientes lóticos continentais brasileiros são escassos (Brassac \& Ludwig 2005), especialmente, em rios que foram submetidos à construção de reservatórios. Considerando o compartimento dos sedimentos aquáticos, inexiste qualquer contribuição, exceto aos raros estudos sobre paleolimnologia realizados em ambientes lênticos no país (Dumont \& Tundisi 1997, Callegaro 1988, 1995, Gianini 1998, Moro \& Bicudo 1998, Moro et al. 2004, Fürstenberger 2001, Souza et al. 2007, Costa 2008).

Apenas quatro trabalhos resumem o conhecimento da diatomoflora do Rio Paranapanema, sendo apenas um taxonômico, que inventariou as diatomáceas do trecho a represar do Reservatório de Rosana, resultando na identificação de 51 táxons (Bicudo et al. 1993). Outro, de cunho ecológico, trata da diversidade das comunidades fitoplanctônicas e inclui ilustrações de 52 táxons de diatomáceas do Rio Tibagi (BittencourtOliveira 2002). Os demais, de caráter eminentemente ecológico, apresentam listas de espécies (Felisberto \& Rodrigues 2005, Henry et al. 2006) ou apenas citações de alguns táxons (Nogueira 2000).

Este trabalho contribui pioneiramente com o levantamento florístico das diatomáceas pertencentes às classes Coscinodiscophyceae e Fragilariophyceae considerando, conjuntamente, a série de reservatórios em cascata do Rio Paranapanema. Representa, ainda,

1. Programa de Pós-graduação em Biodiversidade Vegetal e Meio Ambiente, Instituto de Botânica

2. Instituto de Botânica, Seção de Ecologia, Av. Miguel Stéfano, 3687, 04301-902 São Paulo, SP, Brasil

3. Autor para correspondência: dbicudo@terra.com.br 
contribuição inédita sobre a composição de diatomáceas de sedimentos superficiais de reservatórios em cascata no país. Como o compartimento sedimentar acumula os eventos que ocorreram na bacia de drenagem, os sedimentos representam uma amostra espacial e temporalmente integrada da biodiversidade da área de estudo.

\section{Material e métodos}

O Rio Paranapanema tem suas nascentes localizadas na serra de Paranapiacaba, no Município de Capão Redondo (SP), na área de proteção ambiental da Serra do Mar. Sua bacia hidrográfica localiza-se entre as coordenadas $22-26^{\circ} \mathrm{S}$ e $47-54^{\circ} \mathrm{W}$, estendendose pelo sudoeste do Estado de São Paulo e norte do Estado de Paraná. A área drenada é de 100.800 $\mathrm{km}^{2}$, sendo $47 \%$ em território paulista e $53 \%$ no Paraná. O sistema em cascata de reservatórios do Rio Paranapanema é um dos principais tributários do alto Rio Paraná (Nogueira et al. 2002). O curso principal do rio, em direção leste-oeste, tem extensão total de 929 km, com 570 m de desnível, desenvolvendo-se entre as altitudes 809 e $239 \mathrm{~m}$. A declividade relativamente elevada $\left(0,6 \mathrm{~m} \mathrm{~km}^{-1}\right)$, com restritas planícies de inundação, é uma característica importante do Rio Paranapanema (Agostinho et al. 1995).

O presente estudo abrangeu os cinco reservatórios mais antigos (Jurumirim, Chavantes, Salto Grande, Capivara e Rosana) da série de onze reservatórios em cascata do Rio Paranapanema. O alto Paranapanema é referido ao trecho inicial do rio, incluindo as represas de Jurumirim e Chavantes, o médio Paranapanema abrange o trecho compreendido entre Salto Grande e Capivara e o baixo Paranapanema, a Represa de Rosana.

As amostras de sedimentos superficiais $(10 \mathrm{~cm})$ foram coletadas com Draga Van Veen $\left(308 \mathrm{~cm}^{2}\right)$, entre os meses de janeiro e novembro de 2006. Distribuíramse em três estações de amostragem (zonas de montante, compartimento central ou próximo à desembocadura do tributário principal e barragem) dos reservatórios de Jurumirim, Chavantes, Salto Grande e Capivara e, em duas regiões (montante e barragem), no Reservatório de Rosana (figura 1, tabela 1).

Lâminas permanentes foram preparadas segundo a técnica de oxidação com peróxido de hidrogênio $\left(\mathrm{H}_{2} \mathrm{O}_{2}\right)$ descrita em Battarbee (1986), utilizando Zrax como meio de inclusão. $\mathrm{O}$ exame qualitativo foi, sempre que possível, baseado em análise populacional,

Tabela 1. Localização das estações de amostragem de sedimentos superficiais dos reservatórios em cascata do Rio Paranapanema (SP/PR).

Table 1. Sampling sites from superficial sediments of cascade reservoirs in the Paranapanema River (SP/PR).

\begin{tabular}{|c|c|c|c|c|c|}
\hline Trecho do rio & Reservatório & $\begin{array}{c}\text { Estação de } \\
\text { amostragem }\end{array}$ & Região & Longitude & Latitude \\
\hline $\begin{array}{c}\text { Alto } \\
\text { Paranapanema }\end{array}$ & Jurumirim & $\begin{array}{l}1 \\
2 \\
3\end{array}$ & $\begin{array}{l}\text { Montante } \\
\text { Ribeirão das Posses (braço lateral) } \\
\text { Barragem }\end{array}$ & $\begin{array}{l}38^{\circ} 51^{\prime} 33^{\prime \prime} \mathrm{N} \\
23^{\circ} 22^{\prime} 63^{\prime \prime} \mathrm{S} \\
23^{\circ} 13^{\prime} 43^{\prime \prime} \mathrm{S}\end{array}$ & $\begin{array}{l}94^{\circ} 47^{\prime} 94^{\prime \prime} \mathrm{W} \\
49^{\circ} 00^{\prime} 0,6^{\prime \prime} \mathrm{W} \\
49^{\circ} 133^{\prime} 27^{\prime \prime} \mathrm{W}\end{array}$ \\
\hline & Chavantes & $\begin{array}{l}4 \\
5 \\
6\end{array}$ & $\begin{array}{l}\text { Montante } \\
\text { Compartimento central } \\
\text { Barragem }\end{array}$ & $\begin{array}{l}23^{\circ} 07^{\prime} 57^{\prime \prime} \mathrm{S} \\
23^{\circ} 24^{\prime} 10^{\prime \prime} \mathrm{S} \\
23^{\circ} 08^{\prime} 43^{\prime \prime} \mathrm{S}\end{array}$ & $\begin{array}{l}49^{\circ} 54^{\prime} 58^{\prime \prime} \mathrm{W} \\
49^{\circ} 34^{\prime} 26^{\prime \prime} \mathrm{W} \\
49^{\circ} 42^{\prime} 36^{\prime \prime} \mathrm{W}\end{array}$ \\
\hline $\begin{array}{c}\text { Médio } \\
\text { Paranapanema }\end{array}$ & Salto Grande & $\begin{array}{l}7 \\
8 \\
9\end{array}$ & $\begin{array}{l}\text { Montante } \\
\text { Foz do Rio Pardo } \\
\text { Barragem }\end{array}$ & $\begin{array}{l}22^{\circ} 533^{\prime} 50^{\prime \prime} \mathrm{S} \\
22^{\circ} 54^{\prime} 37^{\prime \prime} \mathrm{S} \\
22^{\circ} 55^{\prime} 11^{\prime \prime} \mathrm{S}\end{array}$ & $\begin{array}{l}49^{\circ} 59^{\prime} 20{ }^{\prime \prime} \mathrm{W} \\
49^{\circ} 58^{\prime} 11^{\prime \prime} \mathrm{W} \\
49^{\circ} 57^{\prime} 52^{\prime \prime} \mathrm{W}\end{array}$ \\
\hline & Capivara & $\begin{array}{l}10 \\
11 \\
12\end{array}$ & $\begin{array}{l}\text { Montante } \\
\text { Compartimento central } \\
\text { (próximo à foz do Rio Tibagi) } \\
\text { Barragem }\end{array}$ & $\begin{array}{l}22^{\circ} 54^{\prime} 01^{\prime \prime} \mathrm{S} \\
23^{\circ} 00^{\prime} 05^{\prime \prime} \mathrm{S} \\
22^{\circ} 39^{\prime} 21^{\prime \prime S}\end{array}$ & $\begin{array}{l}50^{\circ} 47^{\prime} 38^{\prime \prime} \mathrm{W} \\
50^{\circ} 57^{\prime} 16^{\prime \prime} \mathrm{W} \\
51^{\circ} 20^{\prime} 49^{\prime \prime} \mathrm{W}\end{array}$ \\
\hline $\begin{array}{c}\text { Baixo } \\
\text { Paranapanema }\end{array}$ & Rosana & $\begin{array}{l}13 \\
14\end{array}$ & $\begin{array}{l}\text { Montante } \\
\text { Barragem }\end{array}$ & $\begin{array}{l}22^{\circ} 36^{\prime} 06^{\prime \prime} \mathrm{S} \\
22^{\circ} 36^{\prime} 21^{\prime \prime} \mathrm{S}\end{array}$ & $\begin{array}{l}52^{\circ} 09^{\prime} 48^{\prime \prime} \mathrm{W} \\
52^{\circ} 51^{\prime} 50^{\prime \prime} \mathrm{W}\end{array}$ \\
\hline
\end{tabular}




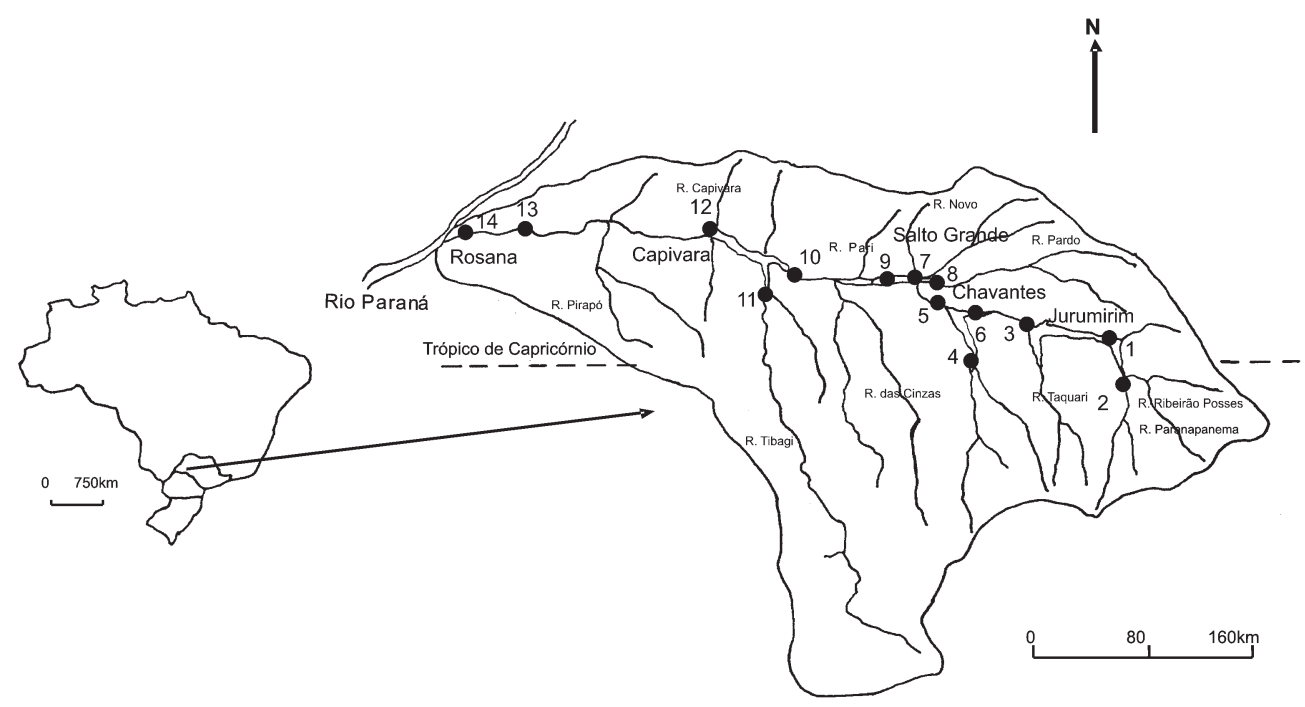

Figura 1. Localização das estações de amostragem nos reservatórios em cascata do Rio Paranapanema. Estações 1 a 6: alto Rio Paranapanema, estações 7 a 12: médio Rio Paranapanema e estações 13 a 14: baixo Rio Paranapanema (modificado de Nogueira et al. 2002).

Figure 1. Sampling sites in the cascade reservoirs in the Paranapanema River. Sampling stations 1 to 6: upper Paranapanema River, 7 to 12: middle Paranapanema River, and 13 to 14: lower Paranapanema River (modified from Nogueira et al. 2002).

de forma a representar a variabilidade morfológica dos indivíduos estudados. A análise foi feita por meio de microscópio óptico binocular Zeiss, Axioscop 2 plus, equipado com contraste-de-fase, câmara-clara e com sistema de captura de imagem. Para fins de distribuição geográfica na bacia hidrográfica do Rio Paranapanema, foram considerados os táxons citados em literatura desde que passíveis de reidentificação.

O sistema de classificação adotado foi o de Round et al. (1990). Alguns gêneros foram propostos em obras mais recentes, tais como Ulnaria (Compère 2001) e Discostella (Houk \& Klee 2004).

As amostras em lâminas permanentes e material seco encontram-se depositadas no acervo do Herbário Científico do Estado "Maria Eneida P. Kauffmann Fidalgo" do Instituto de Botânica da Secretaria do Meio Ambiente do Estado de São Paulo (SP390881 a SP390894).

\section{Resultados e Discussão}

Oestudo florísticodas classes Coscinodiscophyceae e Fragilariophyceae de cinco reservatórios em cascata do Rio Paranapanema permitiu a identificação de 13 táxons, distribuídos em três ordens, quatro famílias e dez gêneros, como seguem:
Coscinodiscophyceae

Thalassiosirales

THALASSIOSIRACEAE

Thalassiosira Cleve

Thalassiosira rudis Tremarin et al., Diatom Research 23(2): 389. 2008.

Figuras 2-4

Valvas circulares, solitárias, superfície valvar plana, ausência de processos (fultoportulae) na região central da valva; aréolas conspícuas de tamanhos irregulares na região central, tornando-se inconspícuas de tamanhos regulares em direção às margens, dispostas radialmente, processos (fultoportulae) formando um anel na margem da valva. Diâmetro: 17,6-35,2 $\mu \mathrm{m}$, processos: 10-11, aréolas em 10 $\mu \mathrm{m}: 11-17$.

Este táxon difere dos demais conhecidos para o gênero pelo complexo padrão de aréolas, ou seja, aréolas conspícuas de tamanhos irregulares na região central, tornando-se inconspícuas e de tamanhos regulares em direção às margens e pela ausência de processos na região central da valva. 
Esta espécie foi recentemente proposta a partir de materiais encontrados em ecossistemas de água doce no Brasil (Reservatórios de Salto Caxias e Faxinal, rios Guaraguaçu e Gonçalves Dias, lagoas Abaeté e Pituaçu) de regiões dos estados do Paraná, Rio Grande do Sul e Bahia (Ludwig et al. 2008).

Thalassiosira é um gênero bem representado em ambiente marinho, porém algumas espécies já foram registradas em ambientes de água doce (Cassie \& Dempey 1980, Torgan \& Santos 2006). Conforme Ferrari \& Ludwig (2007), a ocorrência de espécies de Thalassiosira é escassa em ambientes continentais paranaenses, entretanto, para o Rio Paranapanema, o táxon foi amplamente representado, ocorrendo em $43 \%$ das estações de amostragem. Distribuiu-se nos reservatórios de Jurumirim (estação 3), Chavantes (estações 5 e 6), Salto Grande (estações 7 e 9) e Capivara (estação 10) (tabela 2). Trata-se da primeira citação de ocorrência do táxon para a área de estudo e a segunda ocorrência desta espécie nova.

Material examinado: BRASIL. São PaUlo: Rio Paranapanema, Reservatório de Jurumirim, 24-XI2006, L. Fontana s.n. (SP390883); ParanÁ: Rio Paranapanema, Reservatório de Chavantes, 19IV-2006, L. Fontana s.n. (SP390885, SP390886); Reservatório de Salto Grande, 17-I-2006, L. Fontana s.n.(SP390887, SP390889); Reservatório de Capivara, 17-I-2006, L. Fontana s.n. (SP390890).

\section{STEPHANODISCACEAE}

\section{Cyclotella (Kützing) Brébisson}

Cyclotella meneghiniana Kützing, Bacillarien oder Diatomeen: 50. pl. 30. fig. 68. 1844.

Figuras 5-8

Valvas circulares, superfície valvar lisa a levemente ondulada; área central hialina ou com pontuação granular delicada; estrias alveolares marginais dispostas radialmente, alargando-se em direção à margem valvar. Diâmetro: 6,0-20,9 $\mu \mathrm{m}$; estrias: 8-18 em $10 \mu \mathrm{m}$.

A espécie é comumente encontrada em ambientes lóticos paranaenses (Brassac et al. 1999). Já foi citada para o Reservatório de Rosana por Bicudo et al. (1993), os quais encontraram espécimes com número igual a inferior de estrias. Apresentou ampla distribuição na área de estudo, ocorrendo em todas as estações de amostragem (tabela 2).
Material examinado: BRASIL. São Paulo: Rio Paranapanema, Reservatório de Jurumirim, 24XI-2006, L. Fontana s.n. (SP390881, SP390882, SP390883); PARANÁ: Rio Paranapanema, Reservatório de Chavantes, 19-IV-2006, L. Fontana s.n. (SP390390884, SP390885, SP390886); Reservatório de Salto Grande, 17-I-2006, L.Fontana s.n.(SP390887, SP390888, SP390889); Reservatório de Capivara, 17-I-2006, L. Fontana s.n. (SP390890, SP390891, SP390892); Reservatório de Rosana, 8-VI-2006, L. Fontana s.n. (SP390893, 390894).

\section{Discostella Houk \& Klee}

Chave para os táxons de Discostella

1. Presença de processos marginais conspícuos, estrias irregularmente encurtadas .......................... D.pseudostelligera

1. Presença de processos marginais inconspícuos, estrias de mesmo comprimento D. stelligera

Discostella pseudostelligera (Hustedt) Houk \& Klee, Diatom Research 19(2): 203. 2004 E Cyclotella pseudostelligera Hustedt, Adhandlungen des Naturwissenschaftlichen Verein zu Bremen 31: 591, fig. 1-2. 1939.

Figuras 11-12

Valvas circulares em vista valvar; área central diferenciada da margem valvar, com estria alveolar central, circular, circundada por estrias alveolares encurtadas de tamanhos irregulares, radiadas, formando uma roseta; região marginal com estrias radiadas e presença de processos (fultoportulae) conspícuos na margem valvar. Diâmetro valvar: 7,18,9 $\mu \mathrm{m}$; estrias 13-15 em $10 \mu \mathrm{m}$; processos: 8-9.

Difere de D. stelligera (Cleve \& Grunow) Houk \& Klee por apresentar processos marginais (fultoportulae) bem evidentes ao microscópio óptico. Além disso, D. pseudostelligera possui estrias irregularmente encurtadas, diâmetro valvar menor e valvas menos silicificadas (Haworth \& Hurley 1986).

A espécie já foi citada para o Reservatório de Rosana (Bicudo et al. 1993), ocorrendo, no presente, exclusivamente na montante do Reservatório de Capivara (estação 10).

Material examinado: BRASIL. PARAnÁ: Rio Paranapanema, Reservatório de Capivara, 17-I-2006, L. Fontana s.n. (SP390881). 
Tabela 2. Distribuição dos táxons nas estações de amostragens dos reservatórios em cascata do Rio Paranapanema (SP/PR).

Table 2. Taxa distribution in the sampling sites of the cascade reservoirs in the Paranapanema River (SP/PR).

\begin{tabular}{|c|c|c|c|c|c|c|c|c|c|c|c|c|c|c|}
\hline \multirow{2}{*}{$\frac{\text { Reservatórios }}{\text { Estações de amostragem/táxons }}$} & \multicolumn{3}{|c|}{ Jurumirim } & \multicolumn{3}{|c|}{ Chavantes } & \multicolumn{3}{|c|}{ Salto Grande } & \multicolumn{3}{|c|}{ Capivara } & \multicolumn{2}{|c|}{ Rosana } \\
\hline & 1 & 2 & 3 & 4 & 5 & 6 & 7 & 8 & 9 & 10 & 11 & 12 & 13 & 14 \\
\hline Asterionella formosa var. formosa & & & & & & & & & $\mathrm{x}$ & & $\mathrm{x}$ & & & \\
\hline Aulacoseira granulata var. granulata & $\mathrm{X}$ & $\mathrm{X}$ & $\mathrm{X}$ & & $\mathrm{x}$ & $\mathrm{X}$ & $\mathrm{x}$ & $\mathrm{X}$ & $\mathrm{X}$ & $\mathrm{X}$ & $\mathrm{X}$ & $\mathrm{x}$ & $\mathrm{x}$ & $\mathrm{X}$ \\
\hline A. granulata var. angustissima & & & & & & & & & & $\mathrm{X}$ & $\mathrm{X}$ & $\mathrm{X}$ & & \\
\hline Cyclotella meneghiniana & $\mathrm{X}$ & $\mathrm{X}$ & $\mathrm{X}$ & $\mathrm{X}$ & $\mathrm{x}$ & $\mathrm{X}$ & $\mathrm{x}$ & $\mathrm{x}$ & $\mathrm{X}$ & $\mathrm{X}$ & $\mathrm{x}$ & $\mathrm{X}$ & $\mathrm{x}$ & $\mathrm{X}$ \\
\hline Discostella pseudostelligera & $\mathrm{X}$ & & & & & & & & & & & & & \\
\hline D. stelligera & $\mathrm{X}$ & & $\mathrm{X}$ & & $\mathrm{X}$ & $\mathrm{X}$ & & & $\mathrm{X}$ & & & $\mathrm{X}$ & & \\
\hline Fragilaria capucina & $\mathrm{X}$ & & $\mathrm{x}$ & & & $\mathrm{X}$ & & & $\mathrm{x}$ & & $\mathrm{x}$ & & & \\
\hline Staurosira cf. acutirostrata & & & & & & & & $\mathrm{X}$ & & & & & & \\
\hline Staurosirella martyi & & & & & $\mathrm{X}$ & $\mathrm{X}$ & & $\mathrm{X}$ & & & & & & \\
\hline Staurosirella subcapitata & $\mathrm{X}$ & & & & & & & & & & & & & \\
\hline Synedra goulardii var. goulardii & $\mathrm{X}$ & $\mathrm{x}$ & $\mathrm{X}$ & & $\mathrm{X}$ & & & & & & $\mathrm{X}$ & & $\mathrm{x}$ & $\mathrm{X}$ \\
\hline Thalassiosira rudis & & & $\mathrm{X}$ & & $\mathrm{X}$ & $\mathrm{x}$ & $\mathrm{X}$ & & $\mathrm{x}$ & $\mathrm{X}$ & & & & \\
\hline Ulnaria ulna var. ulna & $\mathrm{X}$ & $\mathrm{x}$ & $\mathrm{X}$ & & $\mathrm{X}$ & & & $\mathrm{X}$ & & & $\mathrm{x}$ & & & \\
\hline Total de táxons / estação de amostragem & 8 & 4 & 7 & 1 & 7 & 6 & 3 & 4 & 6 & 4 & 7 & 4 & 3 & 3 \\
\hline Total de táxons / reservatórios & & 9 & & & 8 & & & 9 & & & 9 & & & 3 \\
\hline
\end{tabular}

Discostella stelligera (Cleve \& Grunow) Houk \& Klee, Diatom Research 19(2): 203. $2004 \equiv$ Cyclotella meneghiniana Kützing var. stelligera Cleve \& Grunow in Cleve, Kongliga SvenskaVetenskaps Akademiens Handlingar 18: pl.5. fig. 63c. 1881.

Figuras 9-10

Valvas circulares em vista valvar; área central diferenciada da margem valvar, com estria alveolar central arredondada, circundada por estrias alveolares de mesmo comprimento, formando uma roseta; estrias alveolares marginais dispostas radialmente. Diâmetro valvar: 8,9-13,1 $\mu \mathrm{m}, 14-17$ estrias em $10 \mu \mathrm{m}$.

Difere de D. pseudostelligera (Hustedt) Houk $\&$ Klee, principalmente, por apresentar processos (fultoportulae) inconspícuos, bem como pela presença de estrias alveolares de mesmo comprimento e valvas mais silicificadas (Haworth \& Hurley 1986).

O táxon já foi citado para o Reservatório de Rosana (Bicudo et al. 1993) e, no presente, distribuiuse nos reservatórios de Jurumirim (estações 1 e 3), Chavantes (estações 5 e 6), Salto Grande (estação 9) e Capivara (estação 12), ocorrendo em $43 \%$ das estações de amostragem (tabela 2).

Material examinado: BRASIL. São Paulo: Rio Paranapanema, Reservatório de Jurumirim, 24-XI2006, L. Fontana s.n.(SP390881, SP390883); PARANÁ: Rio Paranapanema, Reservatório de Chavantes, 19IV-2006, L. Fontana s.n. (SP390885, SP390886); Reservatório de Salto Grande, 17-I-2006, L. Fontana s.n. (SP390889); Reservatório de Capivara, 17-I-2006, L. Fontana s.n. (SP390892). 
Aulacoseirales

\section{AULACOSEIRACEAE}

Aulacoseira Twaites

Chave para os táxons de Aulacoseira

1. Diâmetro valvar superior a $4,0 \mu \mathrm{m}$ A. granulata var. granulata

1. Diâmetro valvar inferior a $2,5 \mu \mathrm{m}$ A. granulata var. angustissima

Aulacoseira granulata (Ehrenberg) Simonsen var. granulata, Bacillaria 2: 58. 1979 = Gallionella granulata Ehrenberg, Abhandlungen der Königlichen Akademie der Wissenschaften zu Berlin 1841: 127. 1843.

Figuras 13-18

Valvas cilíndricas em vista conectival, unidas em cadeias retas por espinhos de ligação curtos e longos; manto ornamentado com estrias areoladas grosseiras, paralelas a levemente oblíquas em relação ao eixo pervalvar. Altura da semicélula: 7,0-24,4

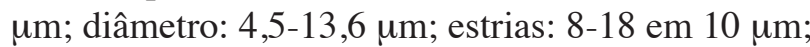
aréolas: 7-9 em $10 \mu \mathrm{m}$.

A população examinada apresentou as características distintivas da espécie, estando plenamente de acordo com a literatura consultada (e.g. Houk 2003, Morandi et al. 2006, Metzeltin \& Lange-Bertalot 2007).

O táxon já foi citado para a área de estudo e, mais especificamente, para o Reservatório de Rosana (Bicudo et al. 1993) e para o Rio Tibagi (BittencourtOliveira 2002). Teve ampla distribuição, ocorrendo nos reservatórios de Jurumirim (estações 1, 2 e 3), Chavantes (estações 5 e 6), Salto Grande (estações 7, 8 e 9), Capivara (estações 10, 11 e 12) e Rosana (estações 13 e 14), ou seja, em $86 \%$ das estações de amostragem (tabela 2).

Material examinado: BRASIL. São PaUlo: Rio Paranapanema, Reservatório de Jurumirim, 24XI-2006, L. Fontana s.n. (SP390882, SP390883); BRASIL. PARANÁ: Rio Paranapanema, Reservatório de Chavantes, 19-IV-2006, L. Fontana s.n. (SP390885, SP390886); Reservatório de Salto Grande, 17-I-2006, L. Fontana s.n. (SP390887, SP390888, SP390889); Reservatório Reservatório de Capivara, 17-I-2006, L. Fontana s.n. (SP390890, SP390891, SP390892); Reservatório de Rosana, 8-VI-2006, L. Fontana s.n. (SP390893, SP390894).
Aulacoseira granulata (Ehrenberg) Simonsen var. angustissima (O. Müller) Simonsen, Bacillaria 2: 58. 1979 = Melosira granulata (Ehrenberg) Ralfs var. angustissima O. Müller, Hedwigia 38: 315, pl.12, fig. 28. 1900.

Figura 19

Valvas cilíndricas em vista conectival, formando cadeias retas, unidas por espinhos de ligação curtos e longos; manto ornamentado por estrias areoladas, delicadas, dispostas paralela a levemente oblíquas em relação ao eixo pervalvar. Comprimento da semicélula: 6,3-11,0 um, diâmetro: 1,4-2,3 um; estrias: 10-12 em $10 \mu \mathrm{m}$; aréolas: 15-20 em $10 \mu \mathrm{m}$.

Essa variedade difere da variedade típica da espécie pelo diâmetro valvar menor e pelas estrias do manto delicadamente areoladas (Hustedt 1927-1930).

A variedade já foi citada para o reservatório de Rosana (Bicudo et al. 1993) e Rio Tibagi (BittencourtOliveira 2002). Apresentou distribuição restrita ao Reservatório de Capivara (tabela 2).

Material examinado: BRASIL. ParanÁ: Rio Paranapanema, Reservatório de Capivara, 17-I-2006, L. Fontana s.n. (SP390890, SP390891, SP390892).

\section{Fragilariophyceae}

Fragilariales

\section{FRAGILARIACEAE}

\section{Asterionella Hassal}

Asterionella formosa Hassal var.formosa, Microscopic Examination of the Water: 10, pl. 2, fig. 5. 1850.

Figuras 20-21

Valvas lineares, heteropolares, extremidades capitadas, distintas do restante da valva; área axial estreita, inconspícua, estrias delicadas de difícil visualização em microscopia óptica. Comprimento: maior do que 46,0 $\mu \mathrm{m}$, largura: 2,7-4,3 $\mu \mathrm{m}$.

Apesar dos espécimes apresentarem as valvas quebradas, a análise de extremidades capitadas de dimensões marcadamente distintas permitiu a identificação dos materiais.

O táxon distribuiu-se apenas em duas estações de amostragens, nos reservatórios de Salto Grande (estação 9) e Capivara (estação 11), ocorrendo em 14\% dos locais amostrados (tabela 2). Trata-se da primeira citação da ocorrência da espécie para a área de estudo. 


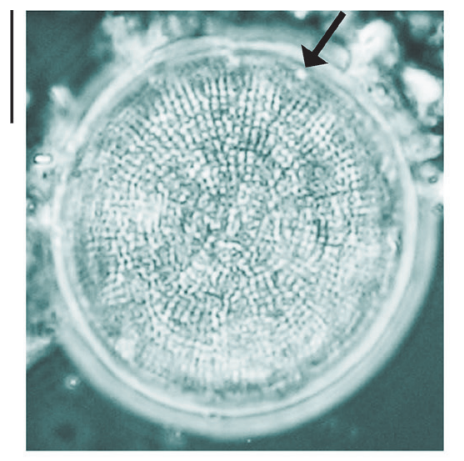

2

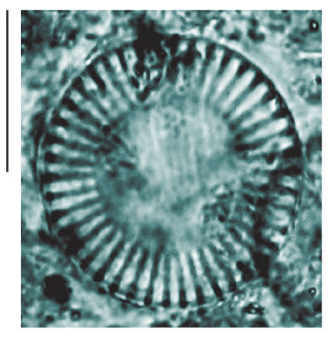

5
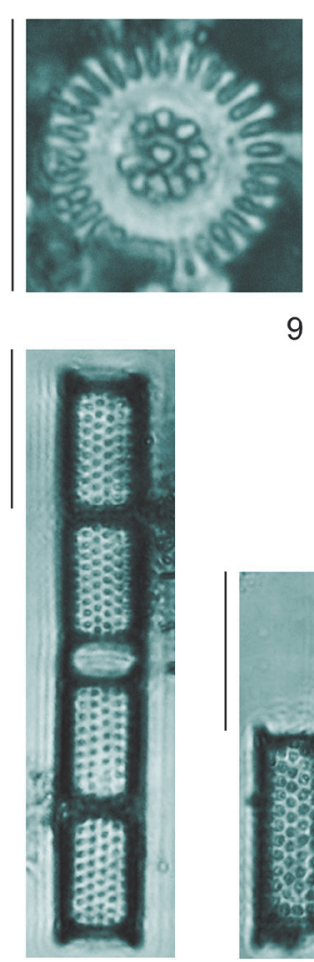

14

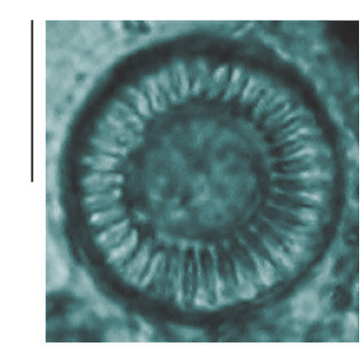

6

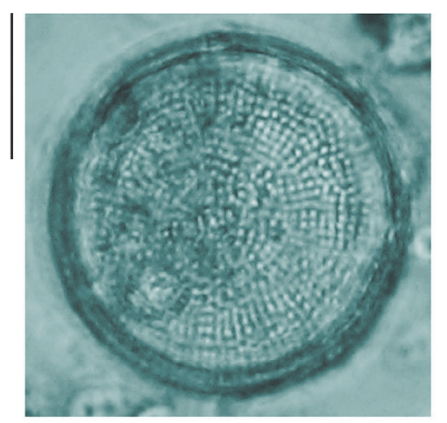

3

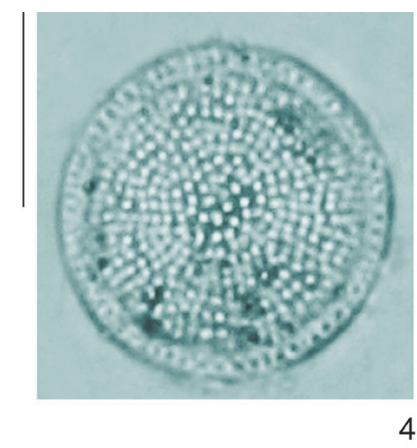

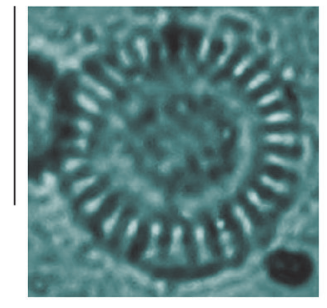

7

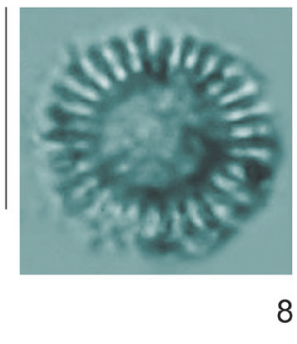

8

9

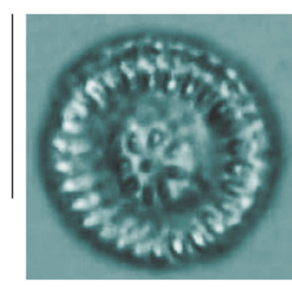

10

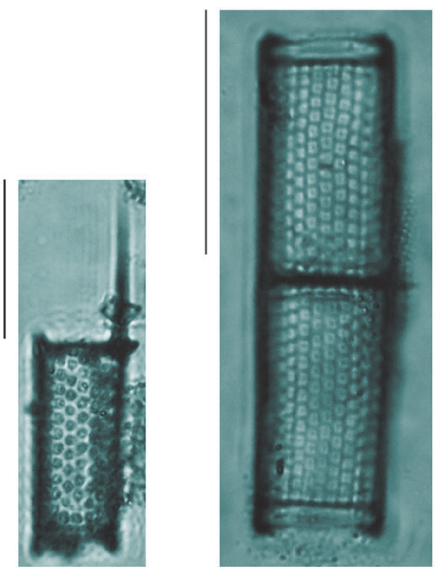

15

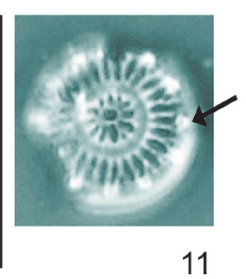

11

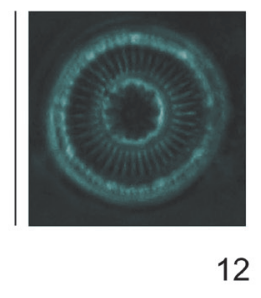

12

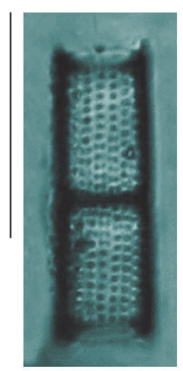

13

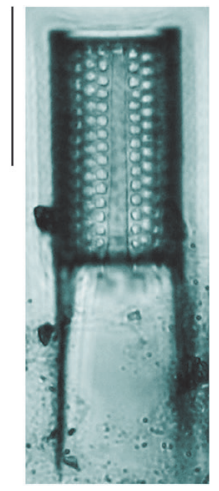

17
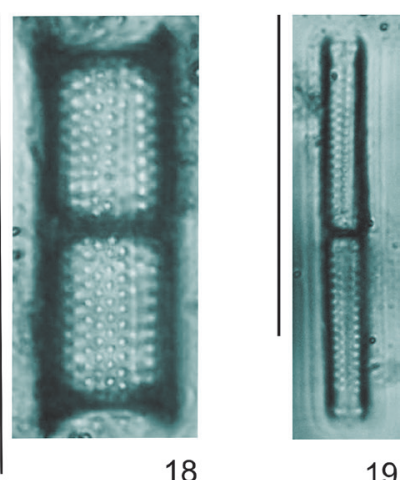

19

Figuras 2-19. Representantes da Classe Coscinodiscophyceae. 2-4. Thalassiosira rudis Tremarin et al. 2. Seta indicando processo marginal (fultoportulae). 5-8. Cyclotella meneghiniana Kützing. 9-10. Discostella stelligera (Cleve \& Grunow) Houk \& Klee. 11-12. Discostella pseudostelligera (Hustedt) Houk \& Klee. 11. Seta indicando processo marginal (fultoportulae). 13-18. Aulacoseira granulata (Ehrenberg) Simonsen var. granulata. 19. A. granulata (Ehrenberg) Simonsen var. angustissima (O. Müller) Simonsen. Barras $=10 \mu \mathrm{m}$.

Figures 2-19. Taxa belonging to class Coscinodiscophyceae. 2-4. Thalassiosira rudis Tremarin et al. 2. Arrow showing marginal process (fultoportulae). 5-8. Cyclotella meneghiniana Kützing. 9-10. Discostella stelligera (Cleve \& Grunow) Houk \& Klee. 11-12. Discostella pseudostelligera (Hustedt) Houk \& Klee. 11. Arrow showing marginal process (fultoportulae), 13-18. Aulacoseira granulata (Ehrenberg) Simonsen var. granulata. 19. A. granulata (Ehrenberg) Simonsen var. angustissima (O. Müller) Simonsen. Scale bars $=10 \mu \mathrm{m}$. 
Material examinado: BRASIL. Paraná: Rio Paranapanema, Reservatório de Salto Grande, 17-I2006, L. Fontana s.n. (SP390889), Reservatório de Capivara, 17-I-2006, L. Fontana s.n. (SP390891).

\section{Fragilaria Lyngbye}

Fragilaria capucina Desmazières in Desmazières, Plantes cryptogames de la France 1(10): 453. 1825.

Figuras 22-24

Valvas lineares a linear-lanceoladas, extremidades subcapitadas; área axial linear, estreita; área central retangular, esterno estreito, linear, estrias robustas, paralelas, intercaladas com as estrias da margem oposta. Comprimento: 22,2-45,7 $\mu \mathrm{m}$, largura: 4,1-7,8 $\mu \mathrm{m}$, estrias: $13-18 \mathrm{em} 10 \mu \mathrm{m}$.

O exame populacional dos materiais não permitiu clara visualização de intumescência bilateral, característica da var. fragilarioides (Grun.) Ludwig \& Flores, ou de intumescência unilateral característica da var. vaucheriae (Kütz.) Lange-Bertalot, razão pela qual optou-se pela identificação em nível específico.

A espécie já foi citada para o Rio Tibagi (Bittencourt-Oliveira 2002). No presente estudo, distribuiu-se nos reservatórios de Jurumirim (estações 1 e 3), Chavantes (estação 6), Salto Grande (estação 9) e Capivara (estação 11), ocorrendo em $36 \%$ das estações amostradas (tabela 2).

Material examinado: BRASIL. São Paulo: Rio Paranapanema, Reservatório de Jurumirim, 24 XI-2006, L. Fontana s.n. (SP390881, SP390883); PARANÁ: Rio Paranapanema, Reservatório de Chavantes, 19-IV-2006, L. Fontana s.n. (SP390886); Reservatório de Salto Grande, 17-I-2006, L. Fontana s.n. (SP390889); Reservatório de Capivara, 17-I-2006, L. Fontana s.n. (SP390891).

\section{Staurosira Ehrenberg}

Staurosira cf. acutirostrata (Metzeltin \& LangeBertalot) Metzeltin \& Lange-Bertalot in Metzeltin, Lange-Bertalot \& García-Rodríguez, Iconographia Diatomologica 15: 229. 2005 E Fragilaria acutirostrata Metzeltin \& Lange-Bertalot, in Lange-Bertalot, Iconographia Diatomologica 5: 89, pl. 1, fig. 18-19, pl. 2, fig. 4. 1998.

Figura 25

Valvas lanceoladas, extremidades valvares projetadas, alongado-subcapitadas; estrias grosseiras, unisseriadas que se estendem pelo manto valvar, disposição alterna, esterno lanceolado. Comprimento: 15,1 $\mu \mathrm{m}$, largura: 4,0 $\mu \mathrm{m}$, estrias: $12 \mathrm{em} 10 \mu \mathrm{m}$.

O único material examinado aproxima-se mais da circunscrição dos exemplares identificados como Fragilaria cf. acutirostrata em Metzeltin \& LangeBertalot (1998: fig. 17, 32), os quais, entretanto, apresentam menor número de estrias (7-9 em $10 \mu \mathrm{m})$. No presente, foi observado esterno lanceolado que, conforme a descrição original da espécie, pode variar de estreito-linear a linear-lanceolado. Muito provavelmente, o exemplar examinado esteja dentro do espectro de variação deste táxon (Staurosira cf.acutirostrata), o qual ainda apresenta circunscrição problemática (Rumrich et al. 2000, Metzeltin et al. 2005).

O táxon ocorreu exclusivamente na foz do Rio Pardo (Represa de Salto Grande, estação 8), sendo citado pela primeira vez para a área de estudo.

Material examinado: BRASIL. PARANÁ: Rio Paranapanema, Reservatório de Salto Grande, 17-I2006, L. Fontana s.n. (SP390888).

Staurosirella Willians \& Round

Chave para os táxons de Staurosirella

1. Extremidades valvares heteropolares Staurosirella martyi

1. Extremidades valvares não heteropolares Staurosirella subcapitata

Staurosirella martyi (Héribaud) Morales \& Manoylov, Diatom Research 21: 354. fig. 34-43. 2006 =Opephora martyi Héribaud, Les Diatomées Fossiles d'Auvergne. Librairie des Sciences Naturelles: 43, pl. 8, fig. 20. 1902.

Figuras 26-27

Valvas lanceoladas, heteropolares, extremidades valvares mais largas truncado-arredondadas a arredondadas, extremidades mais estreitas arredondadas; estrias robustas, paralelas a levemente radiadas; esterno lanceolado. Comprimento: 15-19 $\mu \mathrm{m}$; largura: 5,3-6,4 $\mu \mathrm{m}$; estrias: 6-12 estrias em 10 $\mu \mathrm{m}$.

Morales \& Manoylov (2006) fornecem ampla discussão acerca de $S$. martyi. Segundo estes autores, sob microscopia fotônica, a espécie pode ser distinguida das outras espécies heteropolares do gênero, principalmente, pela presença de valvas fortemente silicificadas e estrias marcadamente refringentes. Os exemplares examinados apresentaram 
tais características e concordaram morfometricamente com a descrição dada por estes autores.

O táxon distribuiu-se restritamente no Reservatório de Chavantes (estações 5 e 6), Salto Grande (estação 8). É a primeira citação da ocorrência da espécie para a área de estudo.

Material examinado: BRASIL. PARAnÁ: Rio Paranapanema, Reservatório de Chavantes, 19-IV2006, L. Fontana s.n. (SP390885, SP390886).

Staurosirella subcapitata (Frenguelli) Morales, Diatom Research 21: 343. 2006 三 Fragilaria pinnata var. subcapitata Frengueli, Revista Chilena de Historia Natural 34: 199, fig. 35, 4. 1930.

Figuras 28-29

Valvas lanceoladas, extremidades valvares rostradas a subrostradas; estrias robustas, paralelas na região central da valva, tornando-se radiadas em direção às margens; esterno linear-lanceolado. Comprimento: 8-28 $\mu \mathrm{m}$; largura: 2,3-5,6 $\mu \mathrm{m}$; estrias: 6-11 em $10 \mu \mathrm{m}$.

Segundo Morales \& Manoylov (2006), Staurosirella subcapitata apresenta grande variação morfológica, caracterizando-se, sob microscopia fotônica, pela forma valvar lanceolada, extremidades acuminadas, rostradas a subrostradas e estrias fortemente refringentes. Ainda conforme esses autores, Staurosirella dubia (Grun.) Morales \& Manoylov é a espécie mais semelhante, diferindo da primeira por apresentar valvas menos lanceoladas e bem menos silicificadas.

A população observada concordou morfometricamente com a descrição original do táxon.

O táxon ocorreu exclusivamente na região de montante do Reservatório de Jurumirim (estação 1), sendo citado pela primeira vez para a área de estudo.

Material examinado: BRASIL. São Paulo: Rio Paranapanema, Reservatório de Jurumirim, 24-XI2006, L. Fontana s.n. (SP390881).

\section{Synedra Ehrenberg}

Synedra goulardii Brébisson ex Cleve \& Grunow var. goulardii, Kongliga Svenska VetenskapsAkademiens Handlingar 17(2): 107, pl.6, fig. 119. 1880.

Figuras 30-32

Valvas linear-lanceoladas, constrição mediana suave a pronunciada; extremidades atenuado- arredondadas, capitadas a subcapitadas; área axial linear, estreita; área central quadrangular, estrias paralelas, opostas. Comprimento: 35,8-60,7 $\mu \mathrm{m}$; largura: 7,3-9,6 $\mu \mathrm{m}$; estrias: 7-14 em $10 \mu \mathrm{m}$.

O táxon já foi citado para o reservatório de Rosana (Bicudo et al. 1993) e, presentemente, ocorreu em $50 \%$ das estações amostradas, distribuindo-se nos reservatórios de Jurumirim (estações 1, 2 e 3), Chavantes (estação 5), Capivara (estação 11) e Rosana (estações 13 e 14) (tabela 2).

Material examinado: BRASIL. São Paulo: Rio Paranapanema, Reservatório de Jurumirim, 24XI-2006, L. Fontana s.n. (SP390881, SP390882, SP390883); PARANÁ: Rio Paranapanema, Reservatório de Chavantes, 19-IV-2006, L.Fontana s.n.(SP390885); Reservatório de Capivara, 17-I-2006, L. Fontana s.n. (SP390891); Reservatório de Rosana, 8-VI-2006, $L$. Fontana s.n. (SP390893, SP390894).

\section{Ulnaria (Kützing) Compère}

Ulnaria ulna (C.L. Nitzsch) Compère var. ulna, in Jahn et al., Studies on diatoms: 100. 2001 $\equiv$ Bacillaria ulna Nitzsch, Neue Schriften der naturforschenden Gesellschaft zu Halle. Hindel's Verlag 3(1): 128, pl. 6. 1817.

Figuras 33-34

Valvas lineares a linear-lanceoladas; extremidades rostradas a subcapitadas; área axial linear; área central retangular delimitada por estrias menores de difícil visualização; estrias paralelas, opostas em relação às da outra margem. Comprimento: superior a $59 \mu \mathrm{m}$; largura: 7,0-7,6 $\mu \mathrm{m}$; estrias: 9-24 em $10 \mu \mathrm{m}$.

A descrição deste táxon baseou-se na análise populacional de várias valvas quebradas.

O táxon já foi citado para o Rio Tibagi (BittencourtOliveira 2002) e, no presente, foi registrado para os reservatórios de Jurumirim (estações 1, 2 e 3), Chavantes (estação 5), Salto Grande (estação 8) e Capivara (estação 11), ocorrendo em $43 \%$ das estações de amostragem (tabela 2).

Material examinado: BRASIL. São PAulo: Rio Paranapanema, Reservatório de Jurumirim, 24XI-2006, L. Fontana s.n. (SP390881, SP390882, SP390883); PARANÁ: Rio Paranapanema, Reservatório de Chavantes, 19-IV-2006, L.Fontana s.n.(SP390885); Reservatório de Salto Grande, 17-I-2006, L. Fontana s.n.(SP390888); Reservatório de Capivara, 17-I-2006, L. Fontana s.n. (SP390891). 

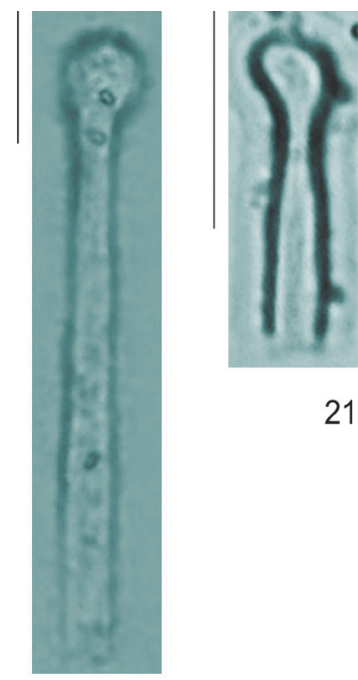

21

20

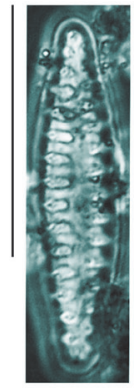

28

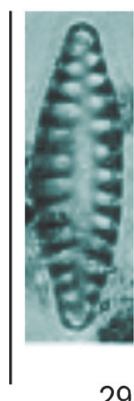

29

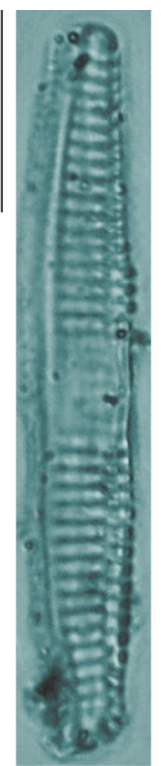

22

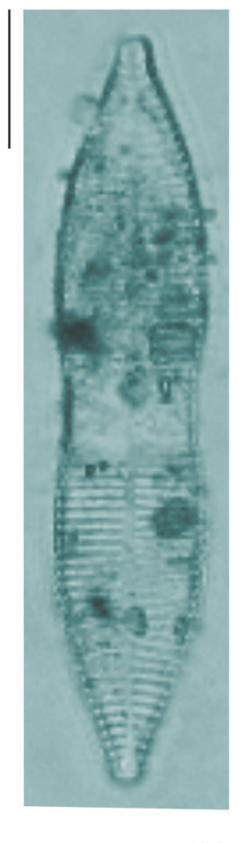

30
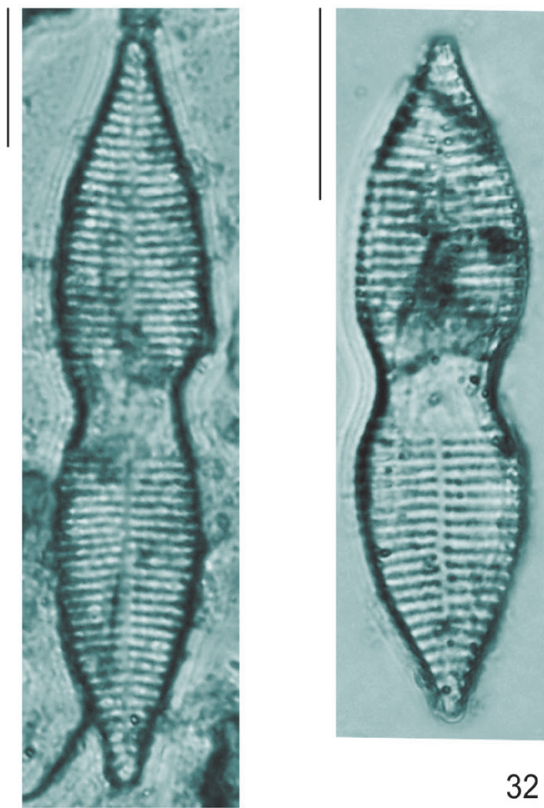

32
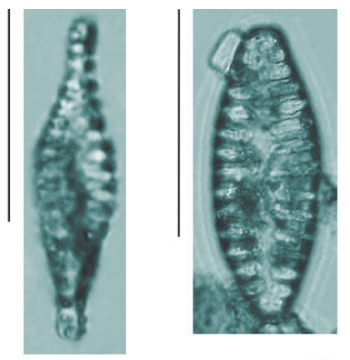

25

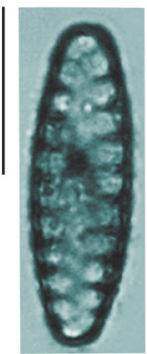

27

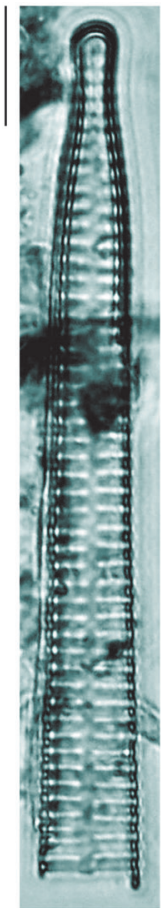

33

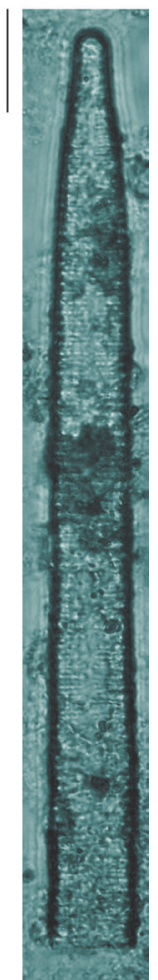

34

Figuras 20-34. Representantes da Classe Fragilariophyceae. 20-21. Asterionella formosa Hassal var. formosa. 22-24. Fragilaria capucina Desmazières. 25. Staurosira cf. acutirostrata (Metzeltin \& Lange-Bertalot) Metzeltin \& Lange-Bertalot. 26-27. Staurosirella martyi (Héribaud) Morales \& Manoylov. 28-29. Staurosirella subcapitata (Frenguelli) Morales. 30-32. Synedra goulardii Brébisson ex Cleve \& Grunow var. goulardii. 33-34. Ulnaria ulna (Nitzsch) Compère var. ulna. Barras $=10 \mu \mathrm{m}$.

Figuras 20-34. Taxa belonging to class Fragilariophyceae. 20-21. Asterionella formosa Hassal var. formosa. 22-24. Fragilaria capucina Desmazières. 25. Staurosira cf. acutirostrata (Metzeltin \& Lange-Bertalot) Metzeltin \& Lange-Bertalot. 26-27. Staurosirella martyi (Héribaud) Morales \& Manoylov. 28-29. Staurosirella subcapitata (Frenguelli) Morales. 30-32. Synedra goulardii Brébisson ex Cleve \& Grunow var. goulardii. 33-34. Ulnaria ulna (Nitzsch) Compère var. ulna. Scale bars $=10 \mu \mathrm{m}$. 
Como considerações finais, destaca-se que as amostragens do compartimento sedimentar permite a obtenção de amostras que integram o espaço e o tempo, sendo assim, ricas de informações sobre a biodiversidade local, difícil de ser obtida a partir de amostras isoladas de assembléias atuais (Bennion 1995). Neste sentido, o presente estudo, realizado a partir de assembléias depositadas nos sedimentos superficiais da série de cinco reservatórios em cascata do Rio Paranapanema, permitiu a identificação de 13 táxons das classes Coscinodiscophyceae e Fragilariophyceae. Em cada reservatório, o número de táxons encontrados variou de 8 a 9 , exceto ao último da série (Reservatório de Rosana), no qual foi bem menor (tabela 2). Em relação à distribuição dos táxons, Aulacoseira granulata (Ehrenberg) Simonsen var. granulata e Cyclotella meneghiniana Kützing apresentaram ampla ocorrência, distribuindose em todos os reservatórios e em 13 e 14 estações de amostragem, respectivamente (tabela 2). As espécies que apresentaram distribuição mais restrita foram Discostella pseudostelligera (Hustedt) Houk \& Klee e Staurosirella subcapitata (Frenguelli) Morales, sendo registradas apenas na estação de montante do Reservatório de Jurumirim. Exceto ao reservatório de Rosana e ao Rio Tibagi, todos os táxons foram registrados pela primeira vez para os demais reservatórios da série em cascata. Ainda, cinco táxons foram citados pela primeira vez para a bacia hidrográfica do Rio Paranapanema: Thalassiosira rudis Tremarin et al., Asterionella formosa Hassal var. formosa, Staurosira cf. acutirostrata (Metzeltin \& Lange-Bertalot) Metzeltin \& Lange-Bertalot, Staurosirella martyi (Héribaud) Morales \& Manoylov e Staurosirella subcapitata (Frenguelli) Morales. Finalmente, este consiste no segundo registro de $T$. rudis Tremarin et al. em âmbito mundial.

\section{Agradecimentos}

Os autores agradecem ao Prof. Dr. Marcos Gomes Nogueira e ao Prof. Dr. Raoul Henry, do Departamento de Zoologia da Universidade Estadual Paulista, Botucatu, pelo inestimável auxílio nas coletas e a "Duke Energy International", pela autorização das coletas. Agradecem, ainda, à FAPESP, Fundação de Amparo à Pesquisa do Estado de São Paulo, pela bolsa em nível de mestrado concedida à primeira autora (processo 05/57399-6) e ao CNPq, Conselho Nacional de Desenvolvimento Científico e Tecnológico (Bolsa concedida a DCB, processo 520745/96-5 to DCB).

\section{Literatura citada}

Agostinho, A.A., Vazzoler, A.E.A.M. \& Thomaz, S.M. 1995. The high river Paraná basin: limnological and ichthyological aspects. In: J.G. Tundisi, C.E.M. Bicudo \& T. Matsumura-Tundisi (eds.). Limnology in Brazil, Academia Brasileira de Ciências e Sociedade Brasileira de Limnologia, Rio de Janeiro, pp. 59-570.

Batarbee, R.W. 1986. Diatoms analysis. In: B.E. Berglund (ed.). Handbook of Holocene Palaeohydrology. John Wiley \& Sons, New York, pp. 527-570.

Bennion, H. 1995. Surface-sediment diatom assemblages in shallow, artificial, enriched ponds and implications for reconstructing trophic status. Diatom Research 10: 1-19.

Bicudo, D.C., Bicudo, C.E.M., Castro, A.A.J. \& PicelliVicentim, M.M. 1993. Diatomáceas (Bacillariophyceae) do trecho a represar do Rio Paranapanema (Usina Hidrelétrica de Rosana), Estado de São Paulo, Brasil. Hoehnea 20: 47-68.

Bittencourt-Oliveira, M.C. 2002. A comunidade fitoplanctônica do rio Tibagi: uma abordagem preliminar de sua diversidade. In: M.E. Medri, E. Bianchini, O.A. Shibatta \& J.A. Pimenta (orgs.). A bacia do rio Tibagi. M.E. Medri, Londrina, pp. 373-402.

Brassac, N.M., Atab, D., Landucci, M., Visinoni, N.D. \& Ludwig, T.A.V. 1999. Diatomáceas cêntricas de rios na região de abrangência da Usina Hidrelétrica de Salto Caxias, PR (bacia do rio Iguaçu). Acta Botanica Brasilica 13: 277-289.

Brassac, N.M. \& Ludwig, T.A.V.2005. Amphipleuraceae e Diploneidaceae (Bacillariophyceae) da bacia do rio Iguaçu, PR, Brasil. Acta Botanica Brasilica 19: 359-368.

Callegaro, V.L.M. 1988. Diatomáceas da turfeira de Águas Claras, planície costeira do rio Grande do Sul: taxonomia, aspectos ecológicos e subsídios à Paleolimnologia. Tese de Doutorado, Universidade Federal do Rio Grande do Sul, Porto Alegre.

Callegaro, V.L.M. 1995. Diatomáceas da Turfeira de Águas Claras, Rio Grande do Sul, Brasil: Gênero Eunotia Ehrenberg. Revista Brasileira de Biologia 55: 369-382.

Cassie, V. \& Dempsey, G.P. 1980. A new freshwater species of Thalassiosira from some oxidation ponds in a new Zealand, and its ultrastructure. Bacillaria 3: 273-292.

Compère, P. 2001. Ulnaria (Kützing) Compère, a new genus name for Fragilaria subgen. Alterasynedra Lange-Bertalot with comments on the typification of Synedra Ehrenberg. In: R. Jahn, J.P. Kociolek, A. Witkowski \& P. Compère (eds.). Lange-Bertalot Festschrift: studies on Diatoms. A.R.G. Gantner, Ruggell, pp. 97-101.

Costa, S.V. 2008. Histórico da eutrofização do Lago das Garças (Parque Estadual das Fontes do Ipiranga, São Paulo) durante o século XX com base no registro 
das diatomáceas em sedimentos. Tese de Doutorado, Instituto de Botânica, São Paulo.

Dumond, H.J. \& Tundisi, J.G. 1997. Preliminary data on the paleolimnology of four lakes. In: J.G. Tundisi \& Y. Saijo (eds.). Limnological studies on the Rio Doce valley lakes, Brazil. Academia Brasileira de Ciências \& Universidade de São Paulo, Rio de Janeiro, pp. 457-474.

Felisberto, S.A. \& Rodrigues, L. 2005. Periphytic community of reservoirs cascade in the Paranapanema river, Brazil. Acta Scientiarum 27: 215-223.

Ferrari, F. \& Ludwig, T.A.V. 2007. Coscinodiscophyceae, Fragilariophyceae e Bacillariophyceae (Achnanthales) dos rios Ivaí, São João e dos Patos, bacia hidrográfica do rio Ivaí, Município de Prudentópolis, PR, Brasil. Acta Botanica Brasilica 21: 421-441.

Fürstenberger,C.B.2001. Interpretações paleolimnológicas do quaternário recente a partir da análise da comunidade de diatomáceas (Bacillariophyceae) no sedimento do Rio Icatu, Município de Xique-Xique, Estado da Bahia, Brasil. Tese de Doutorado, Universidade Estadual Paulista, Rio Claro.

Gianini, C.E.C. 1998. Diatomáceas como indicadoras de acidificação em lagos da Amazônia Central. Dissertação de Mestrado, Instituto Nacional de Pesquisas da Amazônia, Manaus.

Haworth, E.Y.\& Hurley, M.A. 1986. Comparison of the stelligeroid taxa of the centric diatom genus Cyclotella. In: M. Richard (ed.). Proceedings $8^{\text {th }}$ International Symposium of Recent \& Fossil Diatoms. O. Koeltz, Koenigstein, pp. 43-66.

Henry, R., Ushinohama, E. \& Ferreira, R.M.R. 2006. Fitoplâncton em três lagoas marginais ao Rio Paranapanema e em sua desembocadura no Reservatório de Jurumirim (São Paulo, Brasil) durante o periodo prolongado de seca. Revista Brasileira de Botânica 29: 399-414.

Houk, V. 2003. Atlas of freshwater centric diatoms with a brief key and descriptions. Part.I. Melosiraceae, Orthoseiraceae, Paraliaceae and Aulacoseiraceae. Czech Phycology Supplement 1: 1-114.

Houk, V.\& Klee, R. 2004. The stelligeroid taxa of the genus Cyclotella (Kützing) Brébisson (Bacillariophyceae) and their transfer into the new genus Discostella gen. nov. Diatom Research 19: 203-228.

Hustedt, F. 1927-1930. Die Kieselalgen. In: L. Rabenhorst (ed.). Kryptogamen-Flora v. 7. Akademische Verlagsbunchhandlung, Leipzig, pp. 1-920.

Ludwig, T.A.V., Tremarin, P.I., Becker, V. \& Torgan, L.C. 2008. Thalassiosira rudis sp. nov. (Coscinodiscophyceae): A new feshwater species. Diatom Research 23: 389-400.

Metzeltin, D. \& Lange-Bertalot, H. 1998. Tropische Diatomeen in Südamerika I. Iconographia Diatomologica, annotated diatom micrographs v. 5. Koeltz, Stuttgart, pp. 1-695.
Metzeltin, D., Lange-Bertalot, H. \& García-Rodríguez, F. 2005. Diatoms of Uruguay. Iconographia Diatomologica, annotated diatom micrographs v. 15 . Koeltz, Königstein, pp. 1-736.

Metzeltin, D. \& Lange-Bertalot, H. 2007. Tropical diatoms of South America, 2. In: H. Lange-Bertalot (ed.). Iconographia Diatomologica, annotated diatom micrographs v. 18. Koeltz, Stuttgart, pp. 1-877.

Morales, E.A. \& Manoylov, K.M. 2006. Morphological studies on selected taxa in the genus Staurosirella Willians et Round (Bacillariophyceae) from rivers in North America. Diatom Research 21: 343-364.

Morandi, L.L., Ritter, L.M.O., Moro, R.S. \& Bicudo, C.E.M. 2006. Criptógamos do Parque Estadual das Fontes do Ipiranga, São Paulo, SP. Algas, 20: Coscinodiscophyceae. Hoehnea 33: 115-122.

Moro, R.S. \& Bicudo, C.E.M. 1998. Flutuações climáticas no Pleistoceno tardio na Lagoa Dourada, Estado do Paraná, Brasil. Acta Limnologica Brasiliensia 10: 47-58.

Moro, R.S., Bicudo, C.E.M., Mello, M.S., Schimitt, J. 2004. Paleoclimate of the Late Pleistocene and Holocene at Lagoa Dourada, Paraná State, Southern Brazil. Quaternary International 114: 87-99.

Nogueira, M.G. 2000. Phytoplankton composition, dominance and abundance as indicators of environmental compartmentalization in Jurumirim Reservoir (Paranapanema River), São Paulo, Brazil. Hydrobiologia 431: 115-128.

Nogueira, M.G., Jorcin, A., Vianna, N.C.\& Britto, Y.C. 2002. Uma avaliação dos processos de eutrofização nos reservatórios em cascata do rio Paranapanema (SP/PR), Brasil. In: A.C. Fernández \& G.M. Chalar (eds.). El agua em Iberoamérica - de la limnologia a la gestión en Sudamérica. Programa Iberoamericano de Ciencia y Tecnología para el Desarrollo, Buenos Aires, pp. 91-106.

Round, F.E., Crawford, R.M. \& Mann, D.G. 1990. The Diatoms: biology and morphology of the genera. Cambridge University Press, Cambridge.

Rumrich, U., Lange-Bertalot, H. \& Rumrich, M. 2000. Diatomeen der Anden von Venezuela bis Patagonien/ Feuerland. In: H. Lange-Bertalot (ed.). Iconographia Diatomologica 9: 1-672.

Souza, G.S., Koening, M.L., Leça, E.E. \& Coelho, M.P.C.A. 2007. Diatomáceas indicadoras de paleoambientes do Quaternário de Dois Irmãos, Recife, PE, Brasil. Acta Botanica Brasilica 21: 521-529.

Torgan, L.C. \& Santos, C.B. 2006. Thalassiosira weissflogii (Coscinodiscophyceae, Bacillariophyta) em ambientes lacustres na Planície Costeira do sul do Brasil. Iheringia, série Botânica 61: 135-138. 